\title{
Dispersão e predação de sementes de araticum no Cerrado de Mato Grosso, Brasil
}

\author{
Dispersal and predation of araticum seeds in the Cerrado of Mato Grosso, Brazil
}

\author{
Vanessa Golin' Manoel Santos-Filho ${ }^{I I}$ Mônica Josene Barbosa Pereira ${ }^{\text {II, III }}$
}

\section{RESUMO}

\begin{abstract}
Este estudo objetivou caracterizar plantas e frutos de araticum (Annona crassiflora), avaliar a frequência de consumo, predação e dispersão de suas sementes por animais e seus efeitos na germinação, em área de Cerrado, entre fevereiro de 2007 a junho de 2008. Baseado em armadilhas de pegadas, registrou-se a frequência de visitação $(F V)$ e o consumo de frutos por animais, além da ação de insetos em frutos caídos no solo, sob as plantas. Estrumes de antas, Tapirus terrestris, foram amostrados para verificar a frequência de ocorrência (FO) e os danos causados por elas e por insetos. Para analisar a dispersão por antas, foram inseridas miçangas coloridas em frutos maduros caidos sob plantas-mãe. Posteriormente, foi medida a distância entre o local das fezes com miçanga até a planta-mãe da cor específica da miçanga. Realizou-se teste de germinação com sementes de amostras fecais e de frutos intactos. Dentre os animais que se alimentaram dos frutos, antas contribuíram com maior frequência de visitação e consumo de frutos de araticum, o qual representou $54 \%$ de frequência de ocorrência nas amostras fecais e as sementes não foram significativamente danificadas. Besouros (Scarabaeidae) se alimentaram da polpa de frutos e enterraram sementes de araticum próximo da planta-mãe. Insetos curculionideos e euritomideos predaram as sementes de araticum nos frutos. Os danos causados nas sementes por esses insetos foram significativamente maiores que aqueles provocados pelas antas. Foram encontradas duas fezes com miçangas distantes $1,7 \mathrm{~km}$ e $1,8 \mathrm{~km}$ das plantas-mãe, respectivamente. A germinação das sementes foi baixa neste periodo de estudo, no entanto, as antas podem proteger as sementes de insetos predadores, defecando-as intactas para longas distâncias. Assim, T. terrestris é um possivel dispersor,
\end{abstract}

enquanto que curculionídeos e euritomídeos são possíveis predadores de sementes de araticum. Essas relações provavelmente contribuem para o controle da população de araticum no Cerrado brasileiro.

Palavras-chave: Annona crassiflora Mart. (Annonaceae), Tapirus terrestris, dispersores, predadores, Cerrado.

\section{ABSTRACT}

This study aimed to characterize araticum (Annona crassiflora) plants and fruits, and evaluate the frequency of consumption, predation and dispersal of the seeds by animals and its effect on germination, in Cerrado vegetation area, between February 2007 and June 2008. Using track traps installed on araticum plants, the frequency of visitation (FV) and the fruit consumption by the animals was assessed, as well as, the action of insects in fallen fruits under the trees was registered. Sample of tapirs excrement Tapirus terrestris were analyzed to verify the occurrence frequency (FO) and the damage caused on the seeds by tapirs and insects. To analyze the dispersal by tapirs, colored beads were inserted in fallen mature fruits under the parent trees. Then, the distance between the location of the excrements with beads to the parent tree in the specific color of the bead was measured. Germination test was performed with excrement samples and unbroken fruit seeds. Among the animals that fed on fruits, tapirs contributed with the largest frequency of visitation and consumption of araticum fruits, representing 54\% of the frequency occurrence in excrement samples, and the seeds were not damaged significantly. Dung beetles (Scarabaeidae) fed the pulp of the fruit and buried araticum seeds next to the parent tree.

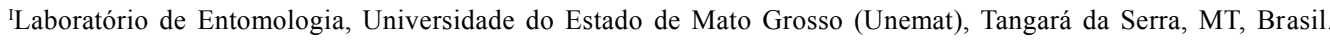

"Programa de Pós-graduação em Ciências Ambientais, Cáceres, MT, Brasil

IIILaboratório de Entomologia, Unemat, Rodovia MT 358, Km 07, CP 287, Bairro Jardim Aeroporto, 78300-000, Tangará da Serra, MT, Brasil. E-mail: monica@unemat.br. Autor para correspondência. 
Curculionid and eurytomid insects predated the araticum seeds on the fruits. The damage caused by these insects in the seeds was significantly higher than those caused by tapirs. Two excrements with beads were found at 1,7 and 1,8km distant from the parent trees, respectively. The seeds had low germinability in this period of study, however the tapirs can protect the seeds of predator insects, defecating the intact seeds for long distances. Thus, T. terrestris is a possible disperser of seeds, whereas curculionids and eurytomids can be considered predators of araticum seeds. These relations probably contribute with the control of araticum population in the Brazilian Cerrado.

Key words: Annona crassiflora Mart. (Annonaceae), Tapirus terrestris, dispersers, predators, Cerrado.

\section{INTRODUÇÃO}

Os vertebrados são responsáveis por 50 a 90\% da dispersão de plantas lenhosas em florestas tropicas (HOWE \& SMALLWOOD, 1982; JORDANO, 2000). No Pantanal, 74\% dos frutos carnosos estão adaptados à dispersão por vertebrados DONATTI et al. (2007). Esses frutos são considerados a base da sustentação para muitos animais no Cerrado, existindo estratégias reprodutivas das plantas e dos consumidores de seus frutos (REYS et al., 2005).

Os frutos de plantas de Annonaceae fazem parte da dieta de muitos vertebrados, os quais podem agir como dispersores ou predadores de sementes (HOWE \& SMALLWOOD, 1982; BODMER, 1989; FRAGOSO \& HUFFMAN, 2000). As sementes podem ser dispersas em três fases: quando os frutos caem no solo, quando são carregadas por vertebrados e quando há remoção por insetos em fezes (VANDER WALL \& LONGLAND, 2004).

As características do fruto do araticum indicam que ele é disperso pela megafauna (GUIMAR ÃES et al., 2008) e a anta (Tapirus terrestris), a qual, hoje em dia, é a única responsável pela dispersão efetiva de um grande número de espécies com frutos grandes e carnosos do Cerrado (DONATTI et al., 2007). Essa espécie foi responsável pelo consumo e possível disseminação de 58 espécies de plantas pertencentes a 23 famílias, incluindo Annonaceae (TÓFOLI, 2006).

Como não existe informação sobre as relações ecológicas entre animais e o araticum no Cerrado, o presente trabalho teve por objetivo caracterizar a dispersão e a predação de sementes de araticum A. crassiflora no Mato Grosso.

\section{MATERIAL E MÉTODOS}

O estudo foi conduzido na área de reserva legal da fazenda Aparecida da Serra (1418'59'S, $57^{\circ} 45^{\prime} 16^{\prime} \mathrm{W}$ e $\left.14^{\circ} 20^{\prime} 23^{\prime} \mathrm{S}, 57^{\circ} 42^{\prime} 39^{\prime \prime} \mathrm{W}\right)$. A área de reserva legal da fazenda possui 4.110ha, constituída por Cerrado sentido restrito, Cerradão e Mata Ciliar. A área selecionada para o estudo foi de 300 ha de Cerrado sentido restrito, por possuir maior adensamento de plantas de araticum em frutificação.

O trabalho de campo foi realizado entre fevereiro e maio de 2007, em que foram monitoradas 95 plantas de araticum em frutificação. Foi registrada a altura de cada planta com auxílio de vara métrica e contados todos os frutos viáveis para o consumo de animais, ou seja, os intactos caídos e aqueles parcialmente danificados por insetos. Do total, 37 frutos maduros de 26 plantas foram sorteados para biometria, os quais foram protegidos ainda verdes com sacos de tecido de voil para evitar a queda ao chão e os danos por animais. Quando atingiram a maturação, foram registrados o diâmetro longitudinal (DL), diâmetro transversal (DT), peso e suas sementes foram contadas e medidos os comprimentos.

A fim de verificar a relação existente entre a altura das plantas e o número de frutos por planta e se existem preferências de animais por plantas com maior disponibilidade de frutos foram realizadas análises de Correlação de Pearson.

Para verificar a frequência de visitação (FV) e a quantidade de frutos consumidos por mamíferos, armadilhas de pegadas foram montadas num raio igual ao da copa da planta. Nas armadilhas, foi retirada a serrapilheira e revolvido o solo para facilitar a impressão e visualização das pegadas. As 95 plantas foram vistoriadas quatro vezes por semana e todos os frutos de cada planta foram contados em todas as visitas para verificar o consumo pelos animais. Os restos de frutos presentes embaixo da planta foram associados com as pegadas nas armadilhas, as quais foram fotografadas e identificadas com o auxílio dos guias de campo.

A observação das aves ocorreu de forma livre, registrando o consumo através de fotografias e filmagem, para identificação da espécie através de bibliografia (SIGRIST, 2006).

Com a finalidade de estimar a distância de dispersão das sementes de araticum por antas, 40 frutos de dez indivíduos, caídos e intactos nas armadilhas de pegadas, foram marcados com miçangas coloridas, sendo uma cor para cada planta monitorada.

Durante o monitoramento, as fezes de antas foram coletadas em latrinas para posterior verificação da presença ou ausência das miçangas ingeridas. A distância de dispersão de sementes por antas foi efetuada com uso de GPS e mediu-se a distância das fezes, contendo as miçangas de cor específica, até a planta-mãe correspondente. 
Todas as sementes de araticum encontradas nas fezes de antas foram contadas e separadas em intactas e danificadas pelas mesmas (amassadas) e pelos insetos (furadas). A frequência de ocorrência (FO) das sementes de araticum em cada amostra fecal foi calculada pelo número de ocorrência das sementes nas amostras fecais, multiplicado por 100 e dividido pelo número de amostras fecais.

Para avaliar se houve diferença significativa entre sementes de araticum danificadas por antas e por insetos, foi utilizado o teste $\mathrm{t}$, com auxílio do programa Systat. As probabilidades obtidas nos testes foram corrigidas pelo método Bonferroni e consideraram-se significativos os valores de probabilidade menores ou iguais a 0,05 .

Os insetos coletados nas fezes de antas e nas plantas de araticum foram levados ao laboratório e identificados.

Para avaliar a germinação das sementes, foram coletadas 20 amostras fecais de antas de 15 latrinas encontradas na área e, como controle, sementes dos frutos maduros de 20 plantas monitoradas. O experimento foi realizado entre maio de 2007 a junho de 2008 , em um viveiro de mudas florestais no município de Tangará da Serra, MT. As sementes foram semeadas (uma em cada recipiente) a dois centímetros de profundidade, em sacos de polietileno, contendo $40 \%$ de substrato Plantmax fibra, $40 \%$ de terra, $10 \%$ de areia e $10 \%$ de esterco de galinha, com cobertura de tela ( $50 \%$ de sombra) e regadas duas vezes ao dia. Foram semeadas cinco sementes de cada amostra fecal e de cada fruto. Consideraram-se como repetições as sementes de cada planta e cada amostra fecal, totalizando 20 repetições por tratamento. As avaliações foram realizadas diariamente, sendo consideradas germinadas quando os cotilédones apareciam sobre a superfície do solo.

\section{RESULTADOS E DISCUSSÃO}

A altura das 95 plantas monitoradas variou de 2 a $5,5 \mathrm{~m}(3,18 \mathrm{~m} \pm 0,86)$. O número de frutos por planta variou de 1 a $22(4,39 \pm 4,12)$ (Tabela 1$)$, não havendo correlação de Pearson significativa $(\mathrm{r}=0,32)$ entre a altura e o número de frutos por planta.

Do total de 417 frutos monitorados, 323 foram considerados viáveis para o consumo dos animais, 37 foram coletados para medidas biométricas (Tabela 1) e 57 estavam secos.

Durante o período de frutificação do araticum, $231(71,52 \%)$ dos 323 frutos viáveis foram consumidos por mamíferos e aves, sendo que 177 foram em março e 54 em abril. Os 92 frutos restantes não foram consumidos, representando $28,48 \%$ do total viável.

As 95 plantas de araticum foram visitadas 188 vezes por animais, que consumiram totalmente $81,6 \%$ dos frutos e parcialmente $59,43 \%$ (Tabela 2), sendo que cada uma foi visitada em média 1,98 vezes, variando de 0 a 15 visitas. Não houve correlação de Pearson significativa $(\mathrm{r}=0,84)$ entre a frequência de visitação dos animais nas plantas e disponibilidade de frutos. Antas visitaram 136 vezes as plantas, consumindo $71,43 \%$ dos frutos individuais disponíveis. Destes, $61,8 \%$ foram consumidos totalmente e 38,2\% parcialmente (Tabela 2).

Obedecendo aos critérios quantitativos de eficiência de dispersão de SCHUPP (1993), que leva em conta o número de sementes dispersas por visita e o número de visitas dos dispersores, considera-se a anta como eficiente para a dispersão do araticum, pois foi a visitante mais frequente nas plantas e consumiu a maioria dos frutos maduros disponíveis.

Verificaram-se poucas visitas e baixo consumo de frutos pelo veado catingueiro (Mazama gouazoubira), lobinho (Cerdocyon thous) e tatu (Dasypus sp.) (Tabela 2). Diferente deste estudo, $\boldsymbol{M}$. gouazoubira consumiu grandes quantidades de frutos de Annonaceae em Floresta Amazônica (BODMER, 1989). Já os dados de lobinho corroboraram os estudos de BUENO \& MOTTA-JÚNIOR (2004) no Cerrado, com baixa ocorrência de itens vegetais, sem a presença de Annonaceae. ANACLETO (2007) não constatou itens

Tabela 1 - Valores mínimo, máximo e a média (com desvio padrão) das características das plantas, frutos e sementes de araticum (Annona crassiflora).

\begin{tabular}{lccc}
\hline Características & Valor mínimo & Valor máximo & Média \pm Desvio padrão \\
\hline Altura planta $(\mathrm{m})$ & 2 & 5 & $3,18 \pm 0,86$ \\
Número frutos por planta & 1 & 22 & $4,39 \pm 4,12$ \\
Peso $(\mathrm{g})$ & 200 & 1.762 & $861,58 \pm 385,35$ \\
Diâmetro $(\mathrm{cm})$ longitudinal & 6,5 & 14,7 & $10,88 \pm 2,15$ \\
Diâmetro $(\mathrm{cm})$ transversal & 8,5 & 14,7 & $11,65 \pm 1,42$ \\
Sementes por fruto & 34 & 153 & $46 \pm 33,25$ \\
Tamanho $(\mathrm{cm})$ sementes & 1 & 2 & 1,5 \\
\hline
\end{tabular}


Tabela 2 - Animais que consumiram frutos de araticum (Annona crassiflora) em área de cerrado sentido restrito, MT. Valores da frequência de visitação (FV) por planta, número total e porcentagem de frutos consumidos e frutos total e parcialmente consumidos.

\begin{tabular}{|c|c|c|c|c|c|c|c|c|}
\hline \multirow{2}{*}{ Animais } & \multicolumn{2}{|c|}{------Visitas-------- } & \multicolumn{6}{|c|}{ 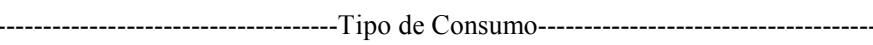 } \\
\hline & FV & $\%$ & Total & $\%$ & Parcial & $\%$ & № total & $\%$ \\
\hline \multicolumn{9}{|l|}{ Mamíferos } \\
\hline Tapirus terrestris & 136 & 72,34 & 102 & 61,82 & 63 & 38,18 & 165 & 71,43 \\
\hline Cerdocyon thous & 2 & 1,06 & 2 & 40,00 & 3 & 60,00 & 5 & 2,16 \\
\hline Dasypus sp. & 4 & 2,13 & 0 & 0,00 & 4 & 100,00 & 4 & 1,73 \\
\hline Mazama gouazoubira & 2 & 1,06 & 1 & 50,00 & 1 & 50,00 & 2 & 0,87 \\
\hline Não identificados & 38 & 20,21 & 20 & 40,82 & 29 & 59,18 & 49 & 21,21 \\
\hline \multicolumn{9}{|l|}{ Aves } \\
\hline Cyanocorax cristatellus & 2 & 1,06 & 0 & 0,00 & 2 & 100,00 & 2 & 0,87 \\
\hline Não identificadas & 4 & 2,13 & 0 & 0,00 & 4 & 100,00 & 4 & 1,73 \\
\hline Total & 188 & 100 & 125 & & 106 & & 231 & 100 \\
\hline
\end{tabular}

vegetais na dieta de tatus no Cerrado. No entanto, pelo menos a espécie D. kappleri pode consumir frutos e engolir sementes inteiras (J.C. Dalponte dados não publicados).

Em 38 ocasiões, não foi possível identificar as espécies de mamíferos por pegadas nas armadilhas, as quais consumiram $21,2 \%$ dos frutos disponíveis (Tabela 2).

Além de mamíferos, aves não identificadas consumiram $1,7 \%$ dos frutos e a gralha-do-campo (Cyanocorax cristatellus) consumiu dois frutos (Tabela 2). SIGRIST (2006) considera essa espécie onívora, dispersando sementes através de regurgitação ou pelas fezes.

Nas 237 amostras fecais de Tapirus terrestris coletadas, o araticum ocorreu em $54 \%$ do total, com 1.531 sementes ingeridas. O número médio de sementes por fezes, considerando apenas as amostras que continham araticum, foi de $12,1 \pm 18,2$, variando de 1 a 96 sementes. Pouco mais da metade $(57,2 \%)$ dessas sementes estava destruída, sendo que, em $3,3 \%$, os danos foram ocasionados durante a passagem pelo tubo digestório das antas. Resultado semelhante foi encontrado por TÓFOLI (2006) em fragmentos de Mata Atlântica, em que $91,2 \%$ das sementes estavam intactas e apenas $8,8 \%$ danificadas pela mastigação de antas.

Dos danos causados às sementes encontradas nas fezes, 53,8\% foram ocasionados por insetos, sendo que a oviposição anterior ao consumo de antas (predação pré-dispersão) foi ocasionada pela vespa Bephratelloides pomorum (Hymenoptera: Eurytomidae), a qual foi encontrada em fruto maduro, representando $0,28 \%$ das ocorrências de insetos em frutos. $\mathrm{O}$ adulto dessa espécie faz oviposição na semente em desenvolvimento e sua larva se alimenta do endosperma até atingir a fase adulta, quando faz um orifício na semente já madura e em seguida constrói uma galeria na polpa do fruto para sair (PEREIRA et al., 2003). Provavelmente, a baixa ocorrência dessa espécie nos frutos observados se deve a sua emergência em frutos antes do amadurecimento.

A predação pós-dispersão, em que o ataque ocorreu nas sementes dos estrumes, foi ocasionado provavelmente pelo besouro Spermologus rufus (Coleoptera: Curculionidae), já que foi observado um indivíduo saindo de dentro da semente.

A diferença na quantidade de sementes intactas e predadas pelos insetos $(\mathrm{n}=128, \mathrm{t}=-0,803$ $\mathrm{P}=1,272$ ) nas fezes não foi significativa. No entanto, as sementes de araticum foram significativamente menos danificadas pelas antas $(\mathrm{n}=128, \mathrm{t}=4,302 \mathrm{P}=0,003)$, quando comparadas com os insetos $(n=128, t=4,919$ $\mathrm{P}=0,003$ ) (Tabela 3).

Apesar de a mortalidade das sementes ter sido menor em latrinas do que nas plantas-mãe, a passagem delas pelo tubo digestório de antas e a contaminação pelas fezes não são suficientes para deter a oviposição e/ou o ciclo de desenvolvimento dos insetos predadores (SILVIUS \& FRAGOSO, 2002; FRAGOSO et al., 2003).

Os curculionídeos $\boldsymbol{S}$. rufus foram observados 92 vezes ( $26,06 \%$ da ocorrência) nos frutos maduros caídos inteiros ou parcialmente consumidos por animais, abaixo da planta-mãe, os quais retiraram as sementes dos frutos para ovipositá-las e as enterraram no solo.

Estudos realizados em florestas tropicais evidenciaram que a predação de sementes prédispersas por insetos foi alta em frutos próximos à planta-mãe, onde há uma maior aglomeração, 
Tabela 3 - Média de sementes intactas e danificadas por antas e insetos, de amostras fecais de antas, e valores do teste $t$ entre as sementes.

\begin{tabular}{llll}
\hline & Intactas x DI $^{1}$ & Intactas x DA $^{2}$ & DI x DA \\
\hline Média intactas & 5,13 & 5,13 & - \\
Média danificadas & 6,44 & 0,40 & 6,44 e 0,40 \\
Valor de $\mathrm{t}$ & $-0,803$ & 4,302 & 4,919 \\
Valor de $\mathrm{p}$ & 1,272 & 0,003 & 0,003 \\
\hline
\end{tabular}

1-DI: Sementes danificadas por insetos.

${ }^{1}$-DA: Sementes danificadas pela anta.

diminuindo à medida que as sementes são levadas por dispersores, reduzindo as sementes viáveis disponíveis para dispersão (JANZEN, 1970; CONNELL, 1971).

Besouros Scarabaeidae também estiveram associados ao araticum como prováveis dispersores: Oxysternon palaemon (Castelnau 1840), Phanaeus palaeno (Blanchard 1843), Diabroctis mirabilis (Harold 1877) e Canthon sp. Eles foram observados 130 vezes, com $36,83 \%$ das ocorrências de insetos encontrados dentro de frutos maduros caídos. Esses animais enterraram sementes sob os frutos, também transportaram e enterraram sementes a distâncias de até $1,2 \mathrm{~m}$ da planta-mãe. São insetos que formam túneis no solo, para onde levam seu alimento, podendo ser eficientes dispersores de sementes (SHEPHERD \& CHAPMAN, 1998).

Outros insetos estiveram presentes em frutos em decomposição, como os das famílias Formicidae $(17,28 \%)$, Staphylinidae $(14,73 \%)$, Tenebrionidae (1,7\%), Erotylidae (0,57\%), Meloidae $(0,28 \%)$, das ordens Isoptera $(1,13 \%)$, Dermaptera $(0,28 \%)$, Hemiptera-Heteroptera $(0,28 \%)$, Diptera $(0,28 \%)$ e Blattodea $(0,28 \%)$.

Dos 40 frutos de araticum monitorados com miçangas coloridas, 35 foram consumidos por antas, confirmado pelas pegadas nas armadilhas. A detecção de marcadores de cores diferentes em cada amostra fecal indicou forrageamento em duas plantas distintas, a distâncias de 1,7 e 1,8km entre o local de deposição das fezes e as plantas-mãe de origem (Figura 1).

Embora 40 frutos tenham sido marcados e a área de estudo pudesse suportar, possivelmente, mais de um indivíduo, considerando que a densidade de antas para o Pantanal varia de 0,31 a 0,71 indivíduos $\mathrm{km}^{-2}$ (TROLLE et al., 2007), foi baixo o número de miçangas encontradas para avaliar a distância de dispersão. Talvez essa área amostral não tenha sido suficiente, considerando a distância linear de até $1,8 \mathrm{~km}$ por um indivíduo para defecação.



Ciência Rural, v.41, n.1, jan, 2011. 
As características dos locais de defecação de T. terrestris, como forma de eliminar sementes intactas, podem favorecer a germinação de $\boldsymbol{A}$. crassiflora e a distribuição de sementes pode gerar adultos dispersos para longe da planta-mãe, o que contribuirá para a perpetuação e a evolução da espécie (FRAGOSO \& HUFFMAN, 2000).

No teste de germinação que durou 414 dias, apenas quatro sementes ( $4 \%$ do total) que passaram pelo tubo digestório de antas germinaram, duas em 89 , uma em 173 e outra em 214 dias. Não houve germinação das sementes controle. Resultado semelhante foi encontrado em Mata Atlântica, em que 3,3\% das sementes de Annona cacans germinaram (TÓFOLI, 2006).

A baixa germinabilidade das sementes de araticum pode estar relacionada às condições inadequadas dos tratamentos, como umidade, luminosidade e temperatura, ou com o embrião da semente, que é imaturo, no qual a dormência, de acordo com DA SILVA et al. (2007), é do tipo morfofisiológica. Nesse caso, é necessário que seja quebrada primeiro a dormência fisiológica antes ou paralelamente à morfológica, a fim de promover o crescimento do embrião.

Assim, a dormência morfológica das sementes de $\boldsymbol{A}$. crassiflora pode ser quebrada pelos animais que as consomem e as defecam intactas, como é o caso de $\boldsymbol{T}$. terrestris, que também pode proteger as sementes de insetos predadores, ao levá-las para longas distâncias.

Estudos como este são importantes para subsidiarem propostas de gestão e manejo de áreas de Cerrado. Faz-se essencial um plano de conservação que considere grandes áreas e a ligação de fragmentos para a dispersão das antas e forrageamento da espécie A. crassiflora, a qual pode depender da distribuição desses animais para aumentar suas chances de sobrevivência.

\section{CONCLUSÃO}

O presente estudo mostrou que a anta (Tapirus terrestris) é responsável pela dispersão efetiva do araticum (Annona crassiflora), enquanto que o besouro Spermologus rufus e a vespa Bephratelloides pomorum são predadores de suas sementes.

\section{AGRADECIMENTOS}

À Marilza da Costa, pelo auxílio de campo. Ao Julio Cesar Dalponte e Jorge José Cherem, pela revisão do manuscrito. Ao Fernando Z. Vaz-de-Mello e Paschoal Grossi, pela identificação dos Scarabaeidae. Ao Sr. Clodoveu Franciosi, por ceder a área de estudo e apoio logístico. Ao PROBIO, pelo suporte financeiro. À CAPES, pela concessão da bolsa de estudo.

\section{REFERÊNCIAS}

ANACLETO, T.C.D.S. Food habits of four armadillo species in the Cerrado area, Mato Grosso, Brazil. Zoological Studies, v.46, p.529-537, 2007. Disponível em: <http:// www.sinica.edu.tw/zool/zoolstud/46.4OnlineFirst/950517.pdf>. Acesso em: 10 jul. 2008.

BODMER, R.E. Frugivory in amazonian Artiodactyla: evidence for the evolution of the ruminant stomach. Journal of Zoology, v.219, p.457-467, 1989. Disponível em: <http:// onlinelibrary.wiley.com/doi/ $10.1111 /$ j. 1469 7998.1989.tb02593.x/abstract>. Acesso em: 23 mar. 2009. doi: 10.1111/j.1469-7998.1989.tb02593.x.

BUENO, A.A.; MOTTA-JÚNOR, J.C. Food habits of two syntopic canids, the maned wolf (Chrysocyon brachyurus) and the crab-eating fox (Cerdocyon thous), in southeastern Brazil. Revista Chilena de Historia Natural, v.35, p.5-14, 2004. Disponível em: $<$ http://www.scielo.cl/scielo.php?pid=S0716$078 X 2004000100002 \&$ script $=$ sci_arttext\&tlng $=\mathrm{en}>$. Acesso em: 26 nov. 2007

CONNELL, J.H. On the role of natural enemies in preventing competitive exclusion in some marine animals and in rain forest tree. In: DEN BOER, P.J.; GRADWELL, P.R. Dynamics of populations. Wageningen: PUDOC, 1971. p.298-312.

DA SILVA, E.A.A. et al. Germination ecophysiology of Annona crassiflora seeds. Annals of Botany, v.99, p.823-830, 2007. Disponível em: <http://aob.oxfordjournals.org/content/99/5/ 823.full.pdf + html>. Acesso em: 22 jun. 2008. doi: 10.1093/ $\mathrm{aob} / \mathrm{mcm} 016$.

DONATTI, C.I. et al. Living in the land of ghosts: fruit traits and the importance of large mammals as seed dispersers in the Pantanal, Brazil. In: DENNIS, A. et al. Frugivory and seed dispersal: theory and applications in a changing world. Wallingford, UK.: Commonwealth Agricultural Bureau International, 2007. p.104-123.

FRAGOSO, J.M.V.; HUFFMAN, J.M. Seed-dispersal and seedling recruitment patterns by the last neotropical megafauna element in Amazonia, the tapir. Journal of Tropical Ecology, v.16, p.369-385, 2000. Disponível em: <http://www.jstor.org/pss/ 3068806>. Acesso em: 08 jun. 2007.

FRAGOSO, J.M.V. et al. Long-distance seed dispersal by tapirs increases seed survival and aggregates tropical trees. Ecology, v.84, p.1998-2006, 2003. Disponível em: <http://www.jstor.org/ pss/3450024>. Acesso em: 08 jun. 2007.

GUIMARÃES, P.R.JR. et al. Seed dispersal anachronisms: rethinking the fruits extinct megafauna ate. PLoS ONE, v.3, p.1-13, 2008. Disponível em: <http://www.plosone.org/article/ info\%3Adoi\%2F10.1371\%2Fjournal.pone.0001745>. Acesso em: 10 fev. 2009. doi: 10.1371/journal.pone. 0001745 .

HOWE, H.F.; SMALLWOOD, J. Ecology of seed dispersal. Annual Review of Ecology and Systematics, v.13, p.201227, 1982. Disponível em: <http://www.jstor.org/pss/2097067>. Acesso em: 10 jun. 2007. 
JANZEN, D.H. Herbivores and the number of tree species in tropical forest. American Naturalist, v.104, p.501-528, 1970. Disponível em: <http://www.jstor.org/pss/2459010>. Acesso em: 08 jun. 2007.

JORDANO, P. Fruits and frugivory. In: FENNER, M. Seeds: the ecology of regeneration in natural plant communities. Wallingford, UK: CAB International, 2000. p.125-66.

PEREIRA, M.J.B. et al. Oviposição da broca-da-semente de graviola Bephratelloides pomorum (Fabricius, 1908) (Hymenoptera: Eurytomidae). Arquivos do Instituto Biológico, v.70, p.221-224, 2003. Disponível em: <http:// www.biologico.sp.gov.br/docs/arq/V70_2/pereira.pdf $>$. Acesso em: 08 jun. 2008 .

REYS, P. et al. Fenologia reprodutiva e disponibilidade de frutos de espécies arbóreas em mata ciliar no rio Formoso, Mato Grosso do Sul. Biota Neotropica, v.5, p.1-10, 2005. Disponível em: <http://www.biotaneotropica.org.br/v5n2/pt/ fullpaper?bn01205022005+pt>. Acesso em: 16 jul. 2007.

SCHUPP, E.W. Quantity, quality and the effectiveness of seed dispersal. In: FLEMING, T.H.; ESTRADA, A. Frugivory and seed dispersal: ecological and evolutionary aspects. Dordrecht, The Netherlands: Kluwer Academic Publishers, 1993. p.15-29.

SHEPHERD, V.E.; CHAPMAN, C.A. Dung beetles as secondary seed dispersers: impact on seed predation and germination. Journal of Tropical Ecology, v.14, p.199-215, 1998. Disponível em: <http://chapmanresearch.mcgill.ca/Pdf/ 93_DungBeetle.pdf>. Acesso em: 15 jul. 2007.
SIGRIST, T. Aves do Brasil: uma visão artística. São Paulo: Fosfertil, 2006. 672p.

SILVIUS, K.M.; FRAGOSO, J.M.V. Pulp handling by vertebrate seed dispersers increases palm seed predation by bruchid beetles in the northern Amazon. Journal of Ecology, v.90, p.10241032, 2002. Disponível em: <http://onlinelibrary.wiley.com/ doi/10.1046/j.1365-2745.2002.00728.x/pdf>. Acesso em: 15 jun. 2007. doi: 10.1046/j.1365-2745.2002.00728.x.

TÓFOLI, C.F. Frugivoria e dispersão de sementes por Tapirus terrestris (Linnaeus, 1758) na paisagem fragmentada do Pontal do Paranapanema, São Paulo. 2006. 98f. Dissertação (Mestrado em Ciências) - Curso de Pós-graduação em Ciências, Instituto de Biociências da Universidade de São Paulo.

TROLLE, M. et al. Brazilian tapir density in the Pantanal: a comparison of systematic camera-trapping and line-transect surveys. Biotropica, v.40, p.211-217, 2007. Disponível em: $<$ http://onlinelibrary.wiley.com/doi/10.1111/j.17447429.2007.00350.x/abstract>. Acesso em: 10 jun. 2008. doi: 10.1111/j.1744-7429.2007.00350.x.

VANDER WALL, S.B.; LONGLAND, W.S. Diplochory: are two seed dispersers better than one? Trends in Ecology and Evolution, v.19 p.155-161, 2004. Disponível em: <http:// www.sciencedirect.com/science? ob=MImg\& imagekey=B6VJ14C3KYND-H-5\&_cdi $=6081 \&$ _user $=8222400 \& \_$pii $=$S0 169534 $703003781 \&$ origin $=$ search $\&$ _zone $=$ rslt_list_item\&_cover Date $=03 \% 2 \mathrm{~F} 31 \% 2 \mathrm{~F} 2004 \&$ sk $=999809996 \& \mathrm{wchp}=\mathrm{dGLzVzz}-$ zSkzS\&md5=d7b618811 f7f938547668f031 cf974b1\&ie=/ sdarticle.pdf $>$. Acesso em: 15 jun. 2007. doi: 10.1016/ j.tree.2003.12.004 